\title{
Internal temperature distributions of interacting and vaporizing droplets
}

\author{
G. Castanet*, A. Labergue, F. Lemoine \\ LEMTA, Nancy-Université, CNRS, 2, Avenue de la forêt de Haye, BP 160, F-54504 Vandœuvre-lès-Nancy, France
}

Keywords:

Droplets

Evaporation

Combustion

Temperature measurements

Convection

\begin{abstract}
A B S T R A C T
A line of mono-sized and periodically spaced droplets is moving in the diffusion flame sustained by the droplet fuel evaporation. The temperature field within the droplets is measured with the help of the twocolor laser-induced fluorescence technique. Experiments are undertaken on droplets made of different fuels including acetone, ethanol, 3-pentanone, $n$-heptane, $n$-decane and $n$-dodecane which have different physical properties such as their volatility and their viscosity. In some cases, the isotherms appear circular and concentric suggesting that only thermal conduction occurs in the droplet. In other cases, measurements show rather significant temperature differences between the leading and the trailing edges of the moving droplets. A simplified model of the heat transfer within the droplet is developed, taking into account both heat conduction and heat advection by the droplet internal fluid circulation. Heat and mass transfer are described in a quasi-steady approach within the framework of the film theory. The internal velocity field is assumed to correspond to the spherical Hill vortex solution, so that the velocity can be related to the stress exerted on the droplet surface. Comparisons between the measurements and the simulations reveal that the heat convection inside interacting droplets is strongly reduced, compared to the model of the isolated droplet.
\end{abstract}

\section{Introduction}

The importance of spray combustion in many industrial applications like turbojets, or direct injection Diesel engines is wellknown. Modeling of spray evaporation is required to optimize combustion chambers performances and to reduce pollutant emissions. As a fuel droplet enters in the overheated environment of the combustion chamber, the droplet is heated and evaporates. The fuel vapor mixes with the atmospheric dioxide, burns and finally heat is released. The accuracy of the simulation tools in this field is highly related to the level of knowledge placed in the description of the elementary phenomena. Spray evaporation has been studied for several decades. In many studies, simplified models for the transient heating of the droplets are used in which the droplet is considered as isolated with uniform transfer coefficients and surface temperature, i.e. spherical symmetry is assumed. Widely used models include [1-3]:

- The 'rapid mixing limit' model which considers that the temperature is uniform in the whole droplet although it may vary in time.

\footnotetext{
* Corresponding author. Tel.: +33 383595646; fax: +33 383595544. E-mail address: guillaume.castanet@ensem.inpl-nancy.fr (G. Castanet).
}

- The 'conduction limit' model which assumes that the heat is transferred in the droplet solely by thermal conduction and that the surface temperature is uniform.

These models are two extremes. In fact, the temperature field within a droplet is not systematically a pure radial one. It results from combined conduction and advection by internal liquid circulation due to the friction at the droplet surface $[4,5]$.

A more physical but still simplified model, is the 'Hill vortex' model, which supposes that the velocity field inside the droplet is given by a Hill vortex. This approach first introduced by Kronig and Brink [6] was used successfully in many research works to describe heat and mass transfers in moving droplets [7-10]. The velocity in the vortex can be related to the friction drag coefficient [7]. However, in the presence of evaporation and wake effects induced by neighboring droplets, no model is currently able to predict accurately the friction force acting on the surface of droplets and very few experimental data are also available in the literature. In an experimental study, Castanet and Lemoine [11] recently measured the temperature field within the ethanol droplets of a monodisperse stream in combustion. They compared experimental temperature distributions with numerical simulations in terms of friction drag coefficient. They found that this latter was significantly reduced (by approximately 90\%) compared to the case of an isolated droplet. However, their analysis was based on a very limited number of experimental cases (only three). This paper is a continuation of this early work. 


\begin{tabular}{|c|c|c|c|}
\hline \multicolumn{2}{|c|}{ Nomenclature } & $t$ & time, $\mathrm{s}$ \\
\hline$a$ & thermal diffusivity, $\mathrm{m}^{2} / \mathrm{s}$ & $Y$ & fuel vapor mass fraction \\
\hline$B_{M}$ & mass transfer Spalding number & & \\
\hline$B_{T}$ & thermal transfer Spalding number & \multicolumn{2}{|c|}{ Greek symbols } \\
\hline C & distance parameter (dimensionless droplet spacing) & $\chi$ & molar fraction \\
\hline$C_{d}$ & drag force coefficient & $\Phi_{\text {vap }}$ & vaporization heat flux, $\mathrm{W}$ \\
\hline$C_{F}$ & friction coefficient & $\Phi_{C}$ & convective heat flux at the liquid-gas interface, $\mathrm{W}$ \\
\hline$C p$ & specific heat, $\mathrm{J} / \mathrm{kg} / \mathrm{K}$ & $\lambda$ & thermal conductivity, $\mathrm{W} / \mathrm{m} / \mathrm{K}$ \\
\hline$D_{g}$ & molecular diffusivity of the vapor, $\mathrm{m}^{2} / \mathrm{s}$ & $\mu$ & dynamic viscosity, $\mathrm{Pa} \mathrm{s}^{-1}$ \\
\hline$K$ & parameter involved in the definition of $C_{F}$ (see (7)) & $\rho$ & density, $\mathrm{kg} / \mathrm{m}^{3}$ \\
\hline Le & Lewis number & $\tau$ & shear stress, $\mathrm{N} / \mathrm{m}^{2}$ \\
\hline$L_{v}$ & latent heat of vaporization, $\mathrm{J} / \mathrm{kg}$ & & \\
\hline$M$ & molar weight, $\mathrm{kg} / \mathrm{mol}$ & \multicolumn{2}{|c|}{ Subscripts } \\
\hline$\dot{m}$ & fuel vapor flow rate, $\mathrm{kg} / \mathrm{s}$ & 0 & isolated droplet without Stefan flow \\
\hline $\mathrm{Nu}$ & Nusselt number & $\mathrm{amb}$ & ambient condition for the calculation of the heat and \\
\hline $\mathrm{Pe}$ & Peclet number & & mass transfer \\
\hline $\operatorname{Pr}$ & Prandtl number & $d$ & droplet \\
\hline$Q_{l}$ & heat flux entering into the droplet, $\mathrm{W}$ & $g$ & gas phase \\
\hline$r_{d}$ & droplet radius, m & inj & condition at the injector exit \\
\hline $\operatorname{Re}$ & droplet Reynolds number & iso & isolated droplet \\
\hline Sh & Sherwood number & $l$ & fuel liquid phase \\
\hline Sc & Schmidt number & $S$ & surface condition \\
\hline$T$ & temperature, $\mathrm{K}$ & $\infty$ & conditions in the free stream far from the droplets \\
\hline
\end{tabular}

It presents a set of experiments obtained in many more aerothermal conditions and with more fuel species. As in Ref. [11], it will be assumed that the friction coefficient of a droplet in interaction can be properly estimated by reducing the friction drag coefficient of the isolated droplet by a factor that depends only on the distance parameter. The purpose of this study is ultimately to determine whether this assumption is consistent with the experimental results.

\section{The Hill vortex model}

Although many studies have reported that the streamlines inside a moving droplet resemble to those of a Hill vortex [12-14], internal circulation can be affected by the Marangoni effect, i.e. gradients of surface tension resulting from temperature variations at the droplet surface can induce thermo-capillary forces at the droplet surface [13]. Additionally, for relatively high Weber numbers, the deformation of the droplet surface can disrupt the structure of the internal flow. In the present study, the Weber number is very low (typical values ranges between 0.7 and 0.9 ), and the aerodynamic deformation of the droplets is not expected to exceed $5 \%$ in terms of eccentricity [15]. Currently, only detailed simulations, including the resolution of the momentum and energy equations inside the droplet, coupled with a calculation of the external flow can take into account these phenomena $[13,16]$. More modestly, in this study, the analysis will be based on the assumption that the streamlines of the internal flow follow the spherical Hill vortex solution (Fig. 1).

The radial and angular component of the velocity can be expressed in the spherical coordinate $(r, \theta, \varphi)$ as

$V_{r}=U_{s}\left(1-r^{2} / r_{d}^{2}\right) \cos \theta$

$V_{\theta}=U_{s}\left(1-2 r^{2} / r_{d}^{2}\right) \sin \theta$,

where $U_{s}$ is the maximum velocity obtained on the surface of the droplet $\left(r=r_{d}\right)$ at the angle $\theta=\pi / 2$.
In the absence of information about the outer flow, the surface temperature of the droplet is assumed to be uniform. The shape of the temperature distribution in the droplet is dependent on the Peclet number $\mathrm{Pe}_{l}$.

$\mathrm{Pe}_{l}=\frac{2 U_{s} r_{d}}{a_{l}}$

When the Peclet number is high, the isotherms coincide with the streamlines of the Hill vortex [17]. Instead, when the Peclet numbers tends to zero, thermal conduction dominates and the temperature field becomes closer to spherical symmetry. For intermediate values, $U_{s}$ is required to determine the shape of the temperature field using the heat transport equation [7]. A friction model is then necessary to determine the shear stress at the droplet surface and hence the internal velocity field [7].

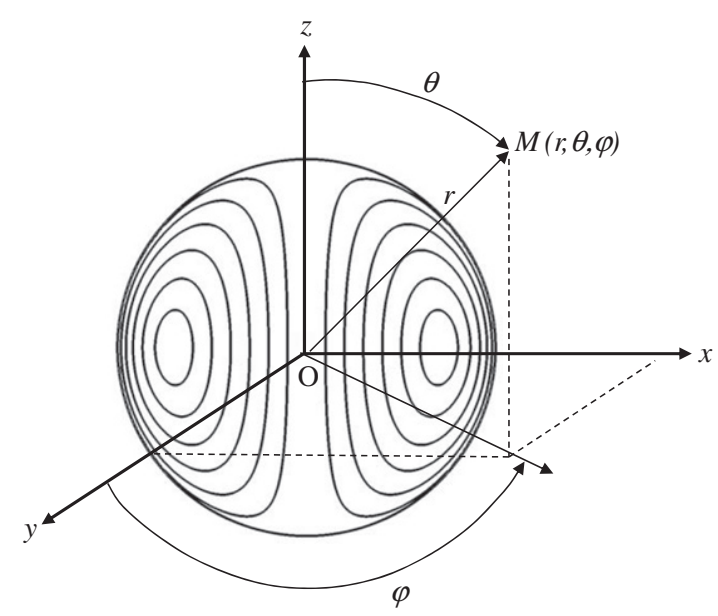

Fig. 1. Streamlines of the spherical Hill vortex. 
In the dimensionless coordinates $\xi=r / r_{d}(t)$ and $\tau=a t / r_{d}^{2}$, the heat transport equation can be expressed as

$\frac{\partial T}{\partial \tau}+\beta \xi \frac{\partial T}{\partial \xi}+\frac{\operatorname{Pe}_{l}}{2} \vec{\nu} \cdot \vec{\nabla} T=\Delta T$

where $\vec{v}=\vec{V} / U_{s}$. The term $\beta=\dot{r}_{d} r_{d} / a_{l}$ accounts for the effect of surface regression on the heat transport and $\dot{r}_{d}=\mathrm{d} r_{d} / \mathrm{d} t$.

The friction force exerted on the droplet surface can be calculated as

$F_{\text {frict }}=2 \pi r_{d}^{2} \int_{0}^{\pi} \tau_{r \theta} \sin ^{2} \theta \mathrm{d} \theta$,

where $\tau_{r \theta}$ is the shear stress on the droplet surface which is continuous through the interface between the liquid and the gas, $\left(\tau_{r \theta}\right)_{g}=\left(\tau_{r \theta}\right)_{l}$. Knowing that the friction coefficient $C_{F}$ is defined by $F_{\text {frict }}=(1 / 2) \rho_{g} \Delta U_{\infty}^{2} S_{d} C_{F}$ and using equations (1) (2) and (5), the maximum liquid velocity $U_{\mathrm{s}}$ can be expressed as [7]

$U_{s}=\frac{1}{32} \frac{\mu_{g}}{\mu_{l}} \operatorname{Re} C_{F} \Delta U_{\infty}$,

where $\Delta U_{\infty}=\left|V_{d}-V_{\infty}\right|$ is the relative gas/droplet velocity and Re the droplet Reynolds number which is defined by $\operatorname{Re}=2 \rho_{\infty} \Delta U_{\infty} r_{d} / \mu_{g}$. In the previous expressions, $\mu_{g}, \rho_{g}$ denotes the viscosity and density of the gaseous phase. They should be calculated taking into account the presence of vapor in the surroundings of the droplet. This is done using the ' $1 / 3$ rule' [18] as described in Section 4.

Based on the friction drag coefficient of a solid sphere [12], $C_{F}=12.69 / \mathrm{Re}^{2 / 3}$, and the results of Renzibulut and Yuen [19] that found that the Stefan flow reduces the drag force by a factor $\left(1+B_{M}\right)$, Abramzon and Sirignano [7] suggested to calculate the friction coefficient as followed:

$C_{F}=\frac{K}{\operatorname{Re}^{2 / 3}\left(1+B_{M}\right)}$

where $K$ is equal to 12.69 for isolated vaporizing droplets.

This expression of $C_{F}$ should be reconsidered for droplets in interactions. In dense spays when the distance between the droplets is comparable with the droplet diameter, the friction coefficient should depend on the droplet concentration in the gas flow. A correction including these effects is missing. Currently, very few results have been reported for dense sprays, this situation being particularly complex to study. Nonetheless, idealized configurations can provide valuable information on these phenomena. In the case of monodisperse droplet streams, droplets are aligned and arranged periodically. The distance parameter $C$, which is usually used to describe the effects of interactions (Fig. 2), is defined as

$C=\frac{L}{2 r_{d}}$,

where $L$ is the droplet spacing.

\section{Experimental set-up}

A linear monodisperse droplet stream is generated by Rayleigh disintegration of a liquid jet with the use of a mechanical vibration obtained by a piezoceramic. For some given frequencies, the liquid jet breaks up into equally spaced and mono-sized droplets (Fig. 2). An electrically heated coil is used to initiate the combustion just after the break-up zone of the liquid jet.

The possibility of mapping the temperature within droplets was extensively described in three previous papers [11,20,21]. Only the main outlines of the measurement technique will be presented here.

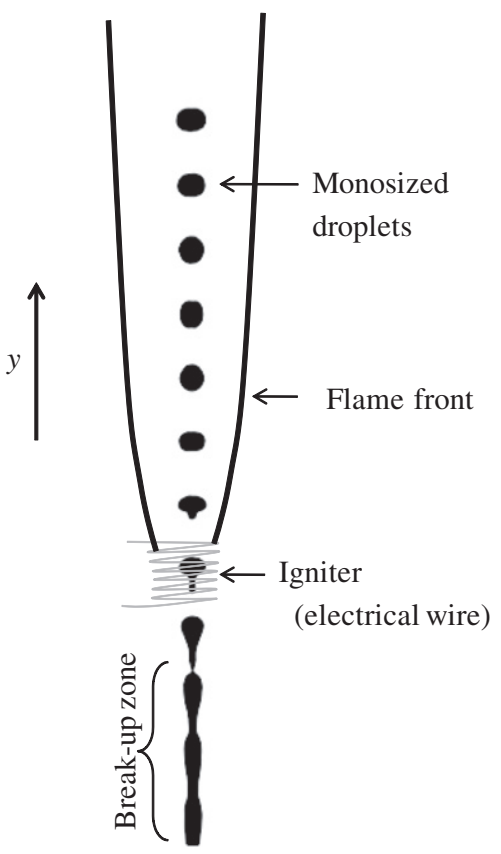

Fig. 2. Monodisperse droplet stream in combustion.

The temperature field is measured with the help of the two-color laser-induced fluorescence technique. The fuel is previously seeded with a low concentration of a fluorescent dye, pyrromethene 597-C8. The fluorescence is induced by the green line of an argon ion laser $(\lambda=514.5 \mathrm{~nm})$. The technique requires two spectral bands of detection with highly different temperature sensitivities. The ratio of the fluorescence intensity measured on the two spectral bands of detection appears to be only temperature dependent and the fluorescence dependencies on tracer concentration, probe volume dimensions, laser intensity and optical layout are removed [22]. For this study, Pyrromethene 597-C8 was chosen over other dyes like Rhodamine B [23] because of its solubility in all the tested fuels (acetone, ethanol, 3 -pentanone, $n$-heptane, $n$-decane, $n$-dodecane) and the temperature sensitivity of its fluorescent signal that is almost unchanged whatever the fuel. Therefore, identical spectral bands of detection [ $540 \mathrm{~nm} ; 560 \mathrm{~nm}$ ] and [590 nm; $610 \mathrm{~nm}$ ] can be selected without detriment to the measurement accuracy. The accuracy of such a temperature measurement is about $\pm 1^{\circ} \mathrm{C}$.

If the probe volume is larger than the droplet to provide a global excitation of all the droplet volume and if the signal is averaged on all the droplet transit in the probe volume, a volume averaged droplet temperature can be obtained $[22,24]$. Conversely, if the probe volume is small compared to the droplet, different zones of the droplet are successively illuminated when the droplets are crossing the laser beams and a local temperature can be obtained [20,21]. Although one laser beam is enough to induce fluorescence, a system of two laser beams crossing at $30^{\circ}$ in the $y-z$ plan was preferred (Fig. 3). This arrangement makes easier the positioning of the droplets in the $z$-direction. The detection optic is positioned so that the beam intersection lies exactly at the middle of the focus plan. The laser beams are focalized to obtain a very narrow measurement volume. Taking into account the lensing effect of the air-droplet interface, the probe volume dimensions are roughly $20 \times 20 \times 34 \mu \mathrm{m}^{3}$. These dimensions appear small enough compared to the droplet size (about $200 \mu \mathrm{m}$ ) to perform spatially resolved explorations of the droplet interior. The injector is shifted progressively by steps of 10 to $20 \mu \mathrm{m}$ in the $x$-direction. For each $x$ position of the injector, droplets are scanned vertically (i.e. along the $y$-axis) as they cross the probe 


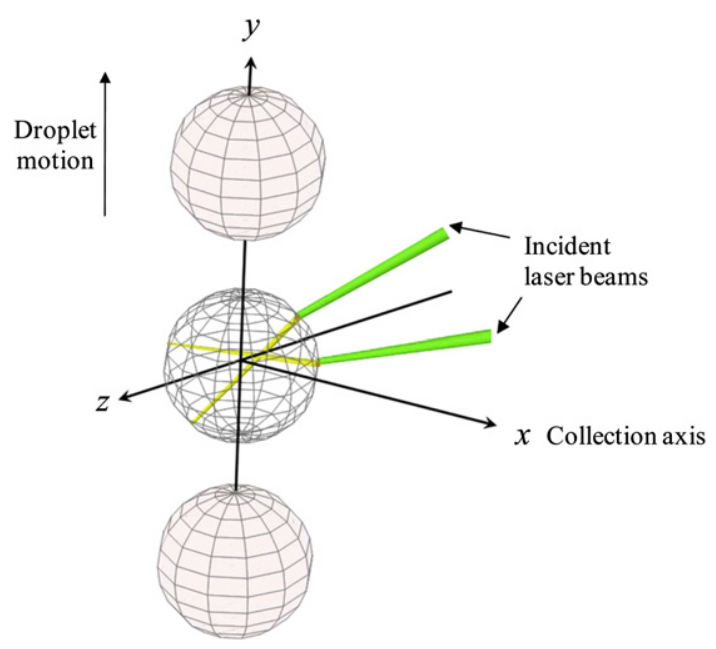

Fig. 3. Optical layout.

volume formed by the intersection of the laser beams. The fluorescence signal is then processed to obtain the evolution of the temperature. The droplet velocity is measured separately with the help of a LDA system. This velocity is required to determine the actual vertical position $y$ of the droplet center at any time during the acquisition. The uncertainty corresponding to the probe volume positioning is estimated around $10 \mu \mathrm{m}$.

Due to the curvature of the droplet, the laser beams are refracted at the droplet surface and the main difficulty is to localize where the measurement zone is exactly located inside the droplet. Given the relative position of the droplet center and the laser beams, geometrical optics with ray tracing can be used to determine the distribution of laser light intensity within the droplet $[20,21]$. In addition with ray tracing, it is also possible to identify the regions in the droplet that are in the field of view of the detection optics. From these calculations, the contribution of any designated region to the total collected fluorescence signal can be quantified. The measured temperature is finally attributed to the points that have the highest contribution to the fluorescence signal and whose the total contribution is equal to $20 \%$ of the global signal. To make a temperature map calculations are repeated for about 200 different positions of the droplet relatively to the laser beam system. Each time, millions of rays are launched to insure a good convergence. An extrapolation between the measurements at these 200 positions provides a temperature map like those presented hereafter for example in Fig. 3.

\section{Description of the experimental temperature maps}

Experiments were undertaken on droplets made of alreadymentioned fuels. These fuels were chosen for their very different volatilities and viscosities (Table 1 ). The droplet velocity is measured by Laser Doppler Anemometry (LDA), which allows evaluating the time elapsed from the injection point. Moderate variations of the droplet velocity were observed, in the order of $0.2-0.4 \mathrm{~m} / \mathrm{s}$, which indicates low drag and friction forces. The droplet size was determined from the volumetric liquid flow rate passing in the injector.

Preliminary numerical simulations (see Sections 4 and 5) reveal very moderate decreases in size, typically less than 3-4 $\mu$ m during the observation time which corresponds to a few milliseconds, even in the case of acetone which is the more volatile fuel. Mass losses due to evaporation are partially compensated by the thermal expansion of the liquid.

Only a limited number of temperature maps are displayed in this article. A summary of the experimental conditions corresponding to
Table 1

Boiling temperature and dynamic viscosity of the tested fuels.

\begin{tabular}{lcl}
\hline & $T_{b}\left({ }^{\circ} \mathrm{C}\right)$ & $\mu\left(\mathrm{Pa} \mathrm{s}^{-1}\right)$ \\
\hline Acetone & 56.5 & $3.98 \times 10^{-4}$ \\
Ethanol & 78.4 & $1.71 \times 10^{-3}$ \\
$n$-heptane & 98.4 & $7.56 \times 10^{-4}$ \\
3-pentanone & 101 & $5.85 \times 10^{-4}$ \\
$n$-decane & 174 & $1.28 \times 10^{-3}$ \\
$n$-dodecane & 215.9 & $4.22 \times 10^{-2}$ \\
\hline
\end{tabular}

these cases is given in Table 2. Few more temperature maps of ethanol exclusively are available in Refs. [11,20,21]. The experimental temperature maps, corresponding to case 1 , are presented in Fig. 4a. In this example, it can be clearly verified that the temperature distribution in moving droplets is generally not purely radial. Significant differences of temperature can be noticed between the leading and the trailing edges of the droplets. Remembering the toroidal shape of the Hill vortex streamlines (Fig. 1), measurements agree qualitatively well with what would be expected from heat advection by a Hill vortex. The surface in contact with hot air is the first region to be heated. Then due to internal circulation, heat is transported to the bottom of the droplet (trailing edge region) and finally it goes up into the core of the droplet. Very similar evolutions have been already described in a previous study in the case of ethanol combusting droplets [11]. The droplet surface is probably cooler near the leading edge. However, it is difficult to conclude since it is not possible to assign a temperature to the regions that are very close to the droplet surface. Laser beams are deflected to the interior of the droplet and the zones that are the most illuminated are not those that are very near the surface [20,21]. Additionally spatial resolution is limited by the size of the beams (about $20 \mu \mathrm{m}^{3}$ ). Case 1 corresponds to one of the highest Peclet numbers of the data set with a value of about 60 (Table 4).

An example where the Peclet number is low (below 20 according to the simulations presented later in Section 4) is depicted in Fig. 4b. It represents the heating of a n-dodecane droplet stream (case 2 ). Temperature gradients are very important because $n$-dodecane has a low volatility compared to the other tested fuels. Isotherms appear circular and concentric. This indicates that convection has a very limited influence. The higher viscosity of $n$-dodecane, the low values of the distance parameter and the droplet velocity limit the internal flow velocity. In this case, a model based solely on heat conduction would be enough to describe correctly the transient heating phase.

Fig. $4 c$ corresponds to the case of an $n$-decane droplet stream (case 3). The temperature evolution appears to be a kind of intermediate between the two previous cases. The Peclet number is about 24 (Table 4). The leading edge, at the top of the droplet, is initially the cooler region (as in case 1 ) but finally the isotherms close in on a cold zone (as in case 2 ) which is slightly off center compared to case 2.

\section{Modeling of the heat transfer within the droplet}

A simplified model of the heat transfer within the droplet is used to account for heat conduction and advection within the

Table 2

Conditions of the injection for the droplet streams presented in Fig. 4 . ( 0 refers to the first measurement point located just above the heated coil used to ignite the flame).

\begin{tabular}{llllll}
\hline & Fuel & $D_{\text {inj }}(\mu \mathrm{m})$ & $V_{0}(\mathrm{~m} / \mathrm{s})$ & $C_{0}$ & $T_{\text {inj }}\left({ }^{\circ} \mathrm{C}\right)$ \\
\hline Case 1 & 3-pentanone & 230 & 9.5 & 4.2 & 23.5 \\
Case 2 & n-dodecane & 180 & 5.3 & 2.3 & 55 \\
Case 3 & n-decane & 190 & 6.7 & 2.7 & 39 \\
\hline
\end{tabular}



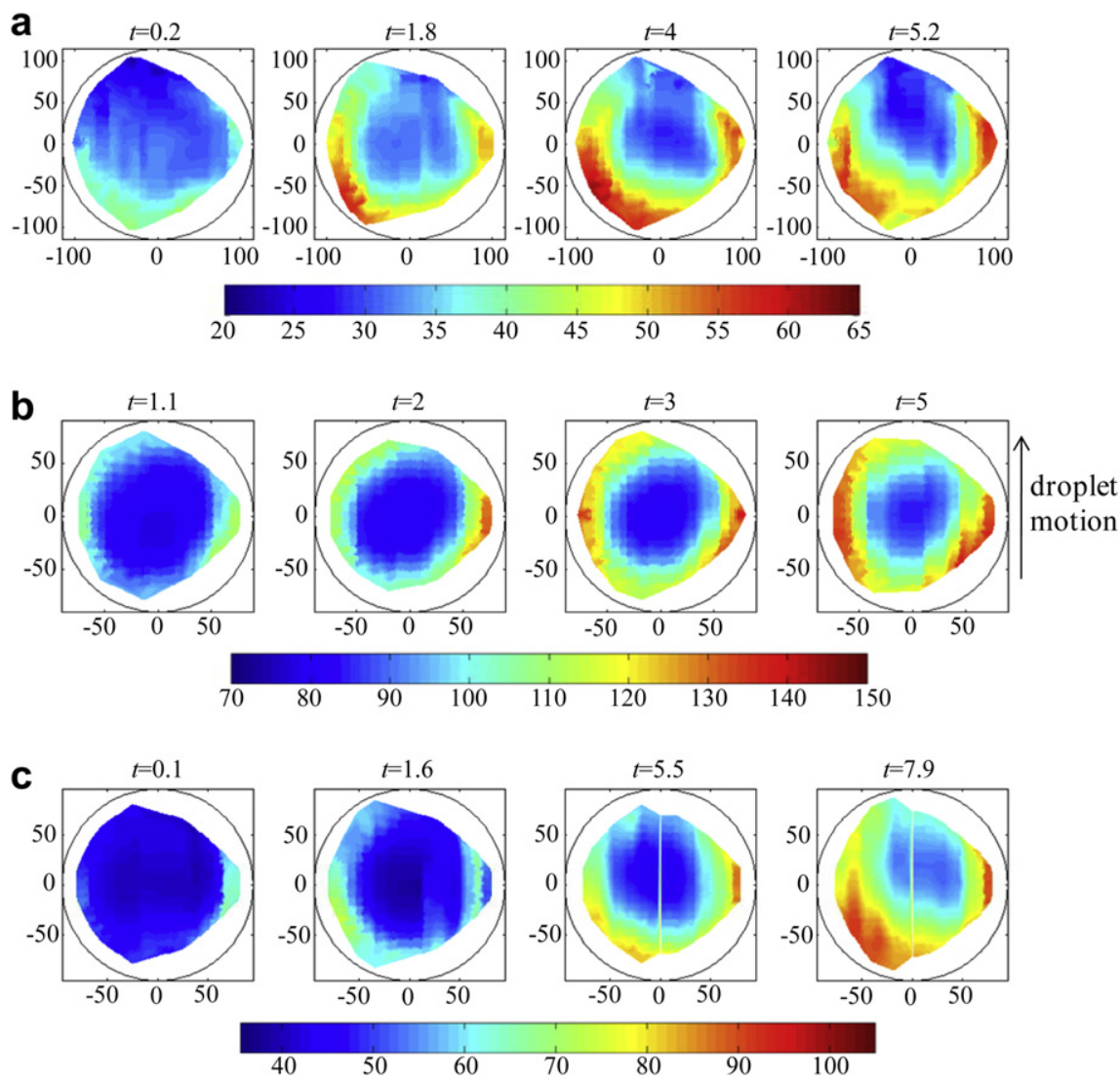

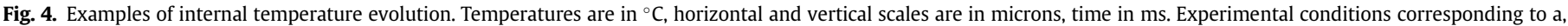
$\mathrm{b}$ and $\mathrm{c}$ also referred to cases 1,2 and 3 in Tables $1-3$. Estimated values of the Peclet number a: $\mathrm{Pe}=55, \mathrm{~b}: \mathrm{Pe}<20$ and $\mathrm{c}: \mathrm{Pe}=24$

droplets. For a given value of the parameter $K$ in the expression of $C_{F}$ (7), the heat transport equation (4) is solved. It is expected that a comparison between the temperature fields obtained theoretically for different values of $K$ and the experimental temperature fields, would eventually make it possible to determine a physical value for $K$ (see Section 6.1). For the resolution, equation (4) is supplemented by the following boundary condition:

$\left.2 \pi r_{d}^{2} \lambda_{l} \int_{0}^{\pi} \frac{\partial T}{\partial r}\right)_{r=r_{d}} \sin \theta \mathrm{d} \theta=Q_{l}(t)$,

where $Q_{l}$ is the heat flux entering into the droplet.

Assuming that the heat of radiation transferred to the droplet can be neglected, the overall energy balance of the evaporation can be summarized as in [25]

$Q_{l}=\Phi_{C}-\Phi_{\mathrm{vap}}$

where $\Phi_{C}$ is the convective heat flux transferred from the gas and $\Phi_{\text {vap }}$ is the heat flux due to the vaporization,

$\Phi_{\mathrm{vap}}=-L_{v} \dot{m}$

$\dot{m}$ being the vapor mass flow rate. $\Phi_{C}$ and $\Phi_{\text {vap }}$ can be derived from the following expressions:

$\Phi_{C}=2 \pi r_{d} \lambda_{g} \mathrm{Nu}\left(T_{\mathrm{amb}}-T_{S}\right)$

$\dot{m}=2 \pi \rho_{g} r_{d} D_{g} B_{M} S h$

where $B_{M}=\left(Y_{S}-Y_{\mathrm{amb}} / 1-Y_{S}\right)$ is the Spalding mass transfer number, $\left.\mathrm{Nu}=2 r_{d} \partial T / \partial r\right)_{r=r_{d}} / T_{\mathrm{amb}}-T_{S}$ is the Nusselt number and
Sh $\left.=-2 r_{d} \partial Y / \partial r\right)_{r=r_{d}} / Y_{S}-Y_{\mathrm{amb}}$ is the Sherwood number. Correlations are required to evaluate $\mathrm{Nu}$ and $\mathrm{Sh}$ in the case of moving and evaporating droplets that are also subject to aerodynamic interactions (equations (16)-(28))

In this simplified approach, the ambient temperature $T_{\mathrm{amb}}$ is not determined using a model. Instead $T_{\mathrm{amb}}$ will be adjusted from the experimental data in a similar way than the parameter $K$ in equation (7) as presented in (5). A sensitivity to this parameter is expected as $T_{\mathrm{amb}}$ controls the convective heat flux and hence the wet-bulb temperature that appears finally at the surface of the droplet.

The fuel vapor mass fraction $Y_{S}$ is determined from the Raoult law, assuming a liquid-vapor equilibrium at the droplet surface

$\chi_{S}=P_{\text {sat }}\left(T_{S}\right) / P$

where $P_{\text {sat }}$ denotes the saturation pressure of the fuel and $\chi_{S}$ the vapor molar fraction at the droplet surface related to $Y_{S}$ by

$Y_{S}=\frac{\chi_{s} M_{v}}{\chi_{S} M_{v}+\left(1-\chi_{S}\right) M_{\mathrm{air}}}$

$M_{v}$ and $M_{\text {air }}$ are respectively the molar mass of the fuel and the air.

To take into account the effect of the droplet motion on the heat and mass transfer in presence of a Stefan flow, Abramzon and Sirignano [7] introduced a diffusion film between the droplet surface and the far conditions in the free flow. The film thickness as defined in the so-called 'film theory' [7], is given by

$\delta_{M}=\frac{2 r_{d} F\left(B_{M}\right)}{\mathrm{Sh}_{0}-2}$ 
$\delta_{T}=\frac{2 r_{d} F\left(B_{T}\right)}{\mathrm{Nu}_{0}-2}$

$B_{T}=C p_{v}\left(T_{\mathrm{amb}}-T_{S}\right) /\left(L_{v}+Q_{l} / \dot{m}\right)$ is the heat transfer Spalding number and $F(B)=(1+B)^{0.7}(\ln (1+B) / B)$.

$\mathrm{Nu}_{0}$ and $\mathrm{Sh}_{0}$ correspond to the Nusselt and Sherwood numbers without Stefan flow. Abramzon and Sirignano [7] suggest the use of the following correlations by Clift et al. [12]

$\mathrm{Nu}_{0}=1+(1+\operatorname{Re} \operatorname{Pr})^{1 / 3} f(\operatorname{Re})$

$\mathrm{Sh}_{0}=1+(1+\operatorname{Re} \mathrm{Sc})^{1 / 3} f(\mathrm{Re})$

with $f(\operatorname{Re})=1$ at $\operatorname{Re} \leq 1$ and $f(\operatorname{Re})=\operatorname{Re}^{0.077}$ at $\operatorname{Re} \leq 400$.

For an isolated droplet, the 'film theory' [7] leads to relatively simple expressions of the Nusselt and Sherwood numbers

$\mathrm{Nu}_{\text {iso }}=\frac{\ln \left(1+B_{T}\right)}{B_{T}}\left(2+\frac{\mathrm{Nu}_{0}-2}{F\left(B_{T}\right)}\right)$

$\mathrm{Sh}_{\text {iso }}=\frac{\ln \left(1+B_{M}\right)}{B_{M}}\left(2+\frac{\mathrm{Sh}_{0}-2}{F\left(B_{M}\right)}\right)$

Combining (20), (21) and the equations (10)-(13), it is possible to demonstrate that $B_{T}$ and $B_{M}$ are linked by the following equation [7]:

$B_{T}=\left(1+B_{M}\right)^{\varphi}-1$

where

$\varphi=\frac{C p_{v}}{C p_{g}} \frac{1}{\mathrm{Le}}$

Le $=\lambda_{g} / C p_{g} \rho_{g} D_{g}$ is the Lewis number.

When droplets are close one another, interactions effects on heat and mass transfer become significant. These effects were studied experimentally by Depredurand et al. [26]. In their experiment, monodisperse droplet streams were injected into a hot air chamber whose temperature can vary up to $400{ }^{\circ} \mathrm{C}$. They tested the same fuel species than those encountered in this study. Their results show that the distance parameter $C$ is not the only parameter to consider in order to describe the effects of interactions on heat and mass transfer. The fuel volatility also plays a significant role. To account for volatility, they introduced a dimensionless parameter $\tau$ which compares the characteristic time of transport through the diffusion film (equations (16) and (17)) and the time separating the passage of two consecutive droplets.

$\tau_{T, M}=\frac{\delta_{T, M} f}{V_{r}}$

$\delta_{T, M}$ is the diffusion film thickness given in (16) and (17) for heat and mass transfers. $V_{r}$ is the radial velocity of the vapor at the droplet surface

$V_{r}=\frac{\dot{m}}{4 \pi \rho_{g} r_{d}^{2}}$

$\tau$ is reduced for volatile products. Indeed a low volatility corresponds to a high resistance to the transfer (high value of $\delta$ ) and a small vapor velocity $V_{r}$. Additionally, $\tau$ decreases when increasing the frequency of the droplets passage. Depredurand et al. [26] proposed an empirical correction of Nusselt and Sherwood numbers based on the parameter $\tau$.

$\mathrm{Nu} / \mathrm{Nu}_{\text {iso }}=\eta\left(\tau_{T}\right)$

$\mathrm{Sh} / \mathrm{Sh}_{\mathrm{iso}}=\eta\left(\tau_{M}\right)$ $\eta(\tau)=\frac{1-\alpha}{(\beta \tau+1)^{\gamma}}+\alpha$

For $\mathrm{Nu} / \mathrm{Nu}_{\text {iso }}$ and $\mathrm{Sh} / \mathrm{Sh}_{\text {iso }}$ of acetone, $\alpha=0.1553, \beta=1.4167$, $\gamma=0.5350$, while for $\mathrm{Sh} / \mathrm{Sh}_{\text {iso }}$ of the other fuel: $\alpha=0.1553$, $\beta=6.5034 \times 10^{-7}, \gamma=54302$ and $\tau \leq 50$.

The resolution of the heat equation is performed by splitting the temperature on the Legendre polynomials base which enables to remove the dependence on the coordinate $\theta[10,11]$. Ten modes appear generally sufficient to get reliable results. An explicit second order Adams-Bashfort scheme is adopted for the time integration while spatial derivatives are approximated by finites differences. The effects of droplets on the gas phase are not considered (one way coupling). These are the main steps of the numerical algorithm:

1- Given $T_{S}$ from the initial condition or from the previous resolution time step, calculate $Y_{S}$ using (14) and (15).

2 - Use the ' $1 / 3$ rule' [18] to obtain the physical properties of the gas phase $\left(\rho_{g}, \lambda_{g}, C p_{g}, D_{g}, \mu_{g}\right)$.

3- Evaluate Pr, Sc and Re. The Reynolds number is calculated with the experimental values of the velocity.

4- Calculate $\mathrm{Nu}_{0}$ and $\mathrm{Sh}_{0}$ using (18) and (19).

5- Find the value of the mass transfer Spalding number $B_{M}$ and then calculate $\mathrm{Sh}_{\mathrm{iso}}, \delta_{M}$ using (16) and (21).

6- Determine $B_{T}$ using (22) and $\mathrm{Nu}_{\text {iso, }}, \delta_{T}$ using (17) and (20).

7- Calculate the vapor mass flow rate $\dot{m}_{\text {iso }}$ of the associated isolated droplet using (13).

8- Since $\dot{m}$ is included in the expressions of $\tau_{M}$ (25), an iterative procedure is required to find mand Sh. $\dot{m}_{\text {iso }}$ is used as an initial guess value in equation (24) to evaluate $\tau_{M}$. A first estimate of Sh is obtained from (27), which leads to a new value of $\dot{m}$ using (13) and $\tau_{M}$ is updated. Iterations continue until Sh has converged with an error of less than $10^{-4}$.

9- Calculate $\tau_{T}$ using (24) and the Nusselt number Nu using (26).

10- Determine $\Phi_{\mathrm{vap}}, \Phi_{C}$ and $Q_{L}$ using (10), (12) and (13).

11- Calculate the friction coefficient $C_{F}$ and the maximal surface velocity $U_{s}$ using (6) and (7).

12- Solve the heat equation (4) to find the distribution of the temperature in the droplet, with the condition that the temperature at the droplet surface must remain uniform. To cope with the integral boundary condition (9), the method suggested by Abramzon and Sirignano [7] is used.

13- Determine the droplet radius at the end of the time step $\Delta t$. Accounting for both the variation of mass due to evaporation and the change of density due to thermal expansion, $\dot{r}_{d}$ can be calculated as followed:

$\dot{r}_{d}=\frac{\dot{m}}{4 \pi r_{d}^{2} \rho_{l}}+\frac{r_{d}\left(\bar{T}_{0}\right)}{\Delta t}\left[\left(\frac{\rho_{l}\left(\bar{T}_{0}\right)}{\rho_{l}\left(\bar{T}_{1}\right)}\right)^{1 / 3}-1\right]$

where $\bar{T}_{0}$ and $\bar{T}_{1}$ are respectively the averaged droplet temperatures at the beginning $t=t_{0}$ and the end of the time step $t=t_{1}$, and $\Delta t=t_{1}-t_{0}$.

14- Return to Step 1 and repeat the calculations for the next time step.

\section{Comparison between the model and the experiments}

To compare experiments with numerical simulations, it is important to apply on the simulation results the same spatial filtering than that resulting from the optical system on the measurements due to the size of the probe volume. Geometrical 


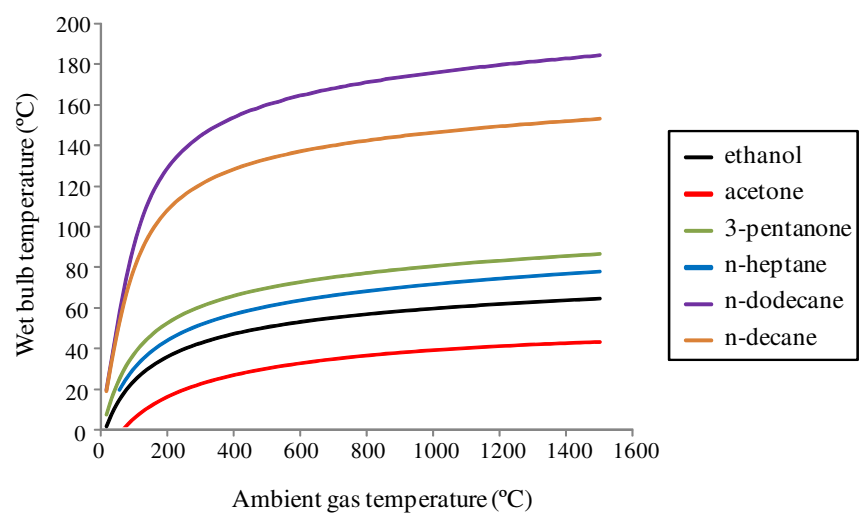

Fig. 5. Wet-bulb temperature as a function of the ambient gas temperature.

optics and ray tracing are used to determine theoretically the fluorescence signal that would have result from the simulated temperature distribution. Theoretical temperature maps are created from the reconstructed fluorescence signal exactly the same manner as described in Section 2 for experimental maps. Additional information about this procedure is available in Refs. [20,21]. Naturally, theoretical maps share some features with their experimental counterparts. For instance, as it can be seen in Figs. 6-8, part of the area close to the surface is not visible and there is a low dissymmetry between the left and the right due to the lensing effect of the droplet surface on the detection.

\subsection{Adjustment of the friction coefficient}

Since no friction model is available for interacting droplets, we propose to reduce the value of the parameter $K$ in the expression (7) of the friction coefficient for an isolated droplet. In this way, the dependencies to the mass transfer Spalding number and the Reynolds number are preserved. This approach seems acceptable since nothing undermines these dependencies in presence of interactions.

Simulations of the droplet heating are performed for several values of $K$ ranging from 0 to 12.69. As a result, the value of $K$ that minimizes the distance $\varepsilon$ between the experimental map $\theta_{\exp }$ and the theoretical maps $\theta_{\text {theo, }}$ can be determined

$\varepsilon=\frac{1}{v_{d}} \int_{v_{d}}\left|\theta_{\text {theo }}-\theta_{\text {exp }}\right| \mathrm{d} v$

In this expression, $v_{d}$ is the part of the droplet volume where the temperature is known and excludes the invisible region near the surface. $\theta$ is the normalized temperature given by
$\theta=\frac{T-T_{\min }}{T_{\max }-T_{\min }}$

where $T_{\min }$ and $T_{\max }$ are the minimum and maximum values of the temperature in the map of interest. To make $\theta$ less dependent on measurement errors, $T_{\min }$ and $T_{\max }$ are calculated on the $5 \%$ points that are the colder or the warmer in the experimental map.

\subsection{Adjustment of the ambient temperature}

Computation of the droplet heating requires having a value for the ambient gas temperature $T_{\mathrm{amb}}$. Note that $T_{\mathrm{amb}}$ is lower than the temperature at the flame front. It can be determined from the wet-bulb temperature $T_{\mathrm{wb}}$ at which the droplet will stabilize after several milliseconds, using equation (22). Fig. 5 shows the correlation between the ambient gas temperature and the wet-bulb temperature for the different fuels of interest. In the temperature range of interest (above $800^{\circ} \mathrm{C}$ ), the ambient gas temperature becomes very sensitive to the wet-bulb temperature. However, as already mentioned, the temperature at the droplet surface is not available from the experiments due to the refraction of the laser beams at the surface (see Section 3). Measurements only give the temperature a few microns below the droplet surface. In addition, it is not clear whether the surface temperature has reached equilibrium at the end of the period corresponding to the measurements. Using the surface temperature instead of the wet-bulb temperature in Fig. 5 could therefore lead to underestimate $T_{\mathrm{amb}}$.

As shown in Fig. 6, the spatial arrangement of the isothermal lines is weakly influenced by the choice of the ambient gas temperature. Although the temperature near the surface is affected by $T_{\mathrm{amb}}$, the distance $\varepsilon$ between experimental and theoretical maps is almost unchanged. Therefore it is possible to perform in first the adjustment of the friction coefficient and subsequently a more refined adjustment of the ambient gas temperature if required. In addition, the vapor concentration is assumed to be zero infinitely far from the droplet surface in the free flow.

\subsection{Determination of the initial condition}

The initial condition is chosen so that the initial temperature field matches the one obtained experimentally at time $t_{0}$. However, as the temperature in the regions close to the surface is unknown, this leaves few degrees of freedom in setting the initial surface temperature. Additionally, it is assumed that the heating remains restricted to the regions near the droplet surface in the initial instant. Therefore, spherical temperature distributions are chosen as initial conditions for the simulations.

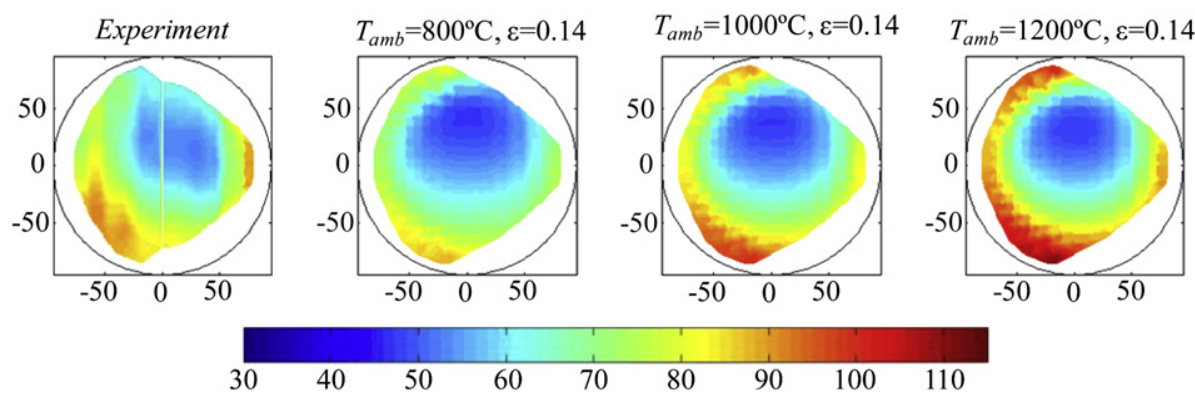

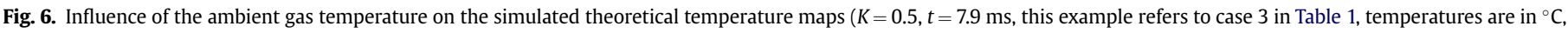
horizontal and vertical scales are in microns). 

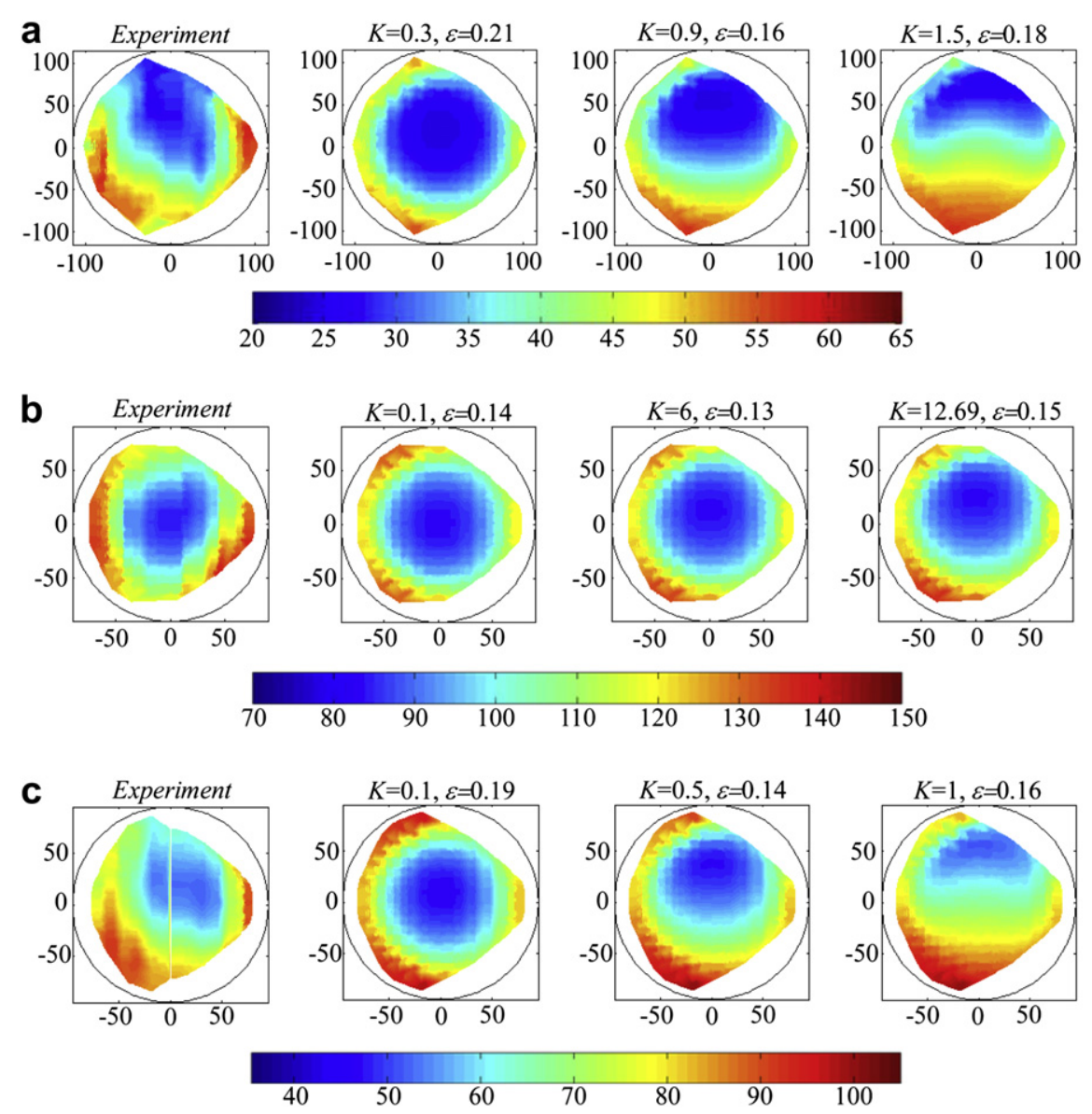

Fig. 7. Adjustment of the friction coefficient. Sensitivity to the parameter $K$ (results corresponding to the same cases as in Fig. 4).

$T_{0}(r)=\left\{\begin{array}{l}T_{c}, r \leq r_{d c} \\ A\left(r-r_{d c}\right)^{2}+T_{c}, \quad r_{d c} \leq r \leq r_{d}\end{array}\right.$

According to (32), the initial temperature $T_{0}$ is uniform in the center of the droplet up to a certain radius $r_{d c}$, then it increases as a parabola up to the surface.
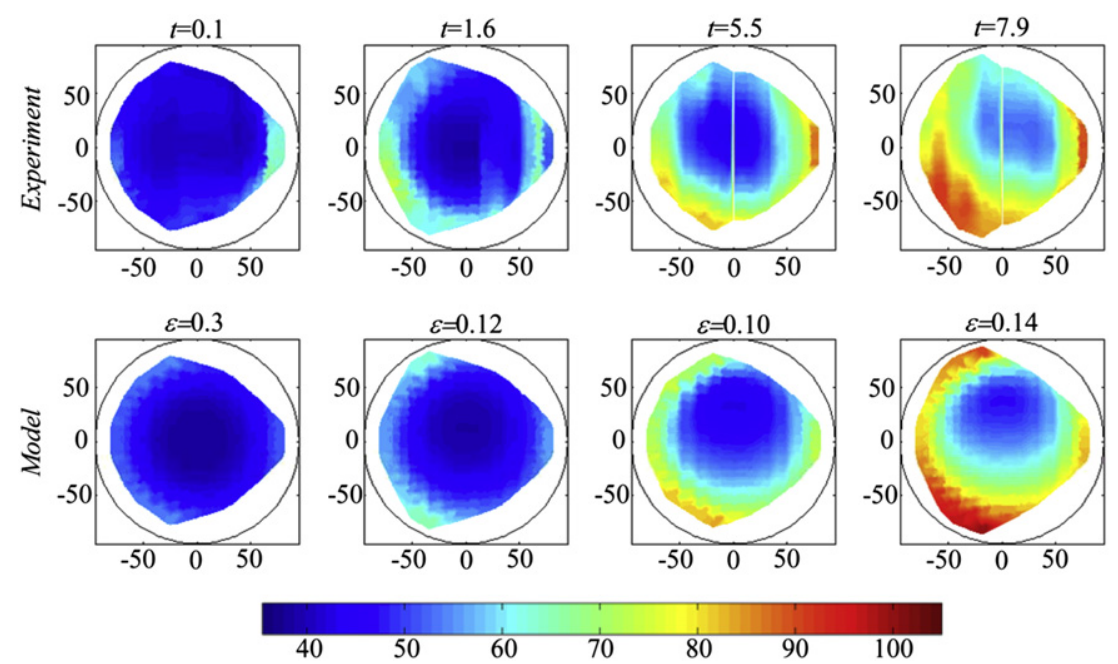

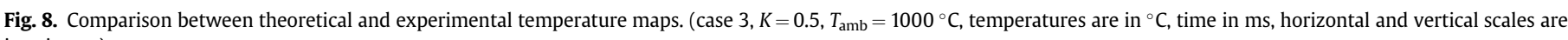
in microns). 
Table 3

Initial and external conditions used to obtain the theoretical temperature maps displayed in Fig. 7.

\begin{tabular}{lllcl}
\hline & $T_{C, 0}\left({ }^{\circ} \mathrm{C}\right)$ & $T_{m, 0}\left({ }^{\circ} \mathrm{C}\right)$ & $T_{S, 0}\left({ }^{\circ} \mathrm{C}\right)$ & $T_{\mathrm{amb}}\left({ }^{\circ} \mathrm{C}\right)$ \\
\hline Case 1 & 24 & 42 & 70 & 1400 \\
Case 2 & 80 & 93 & 111 & 1400 \\
Case 3 & 38 & 50 & 60 & 1000 \\
\hline
\end{tabular}

as time increases, more important differences arise between the temperatures calculated with different values of $K$. The surface temperature $T_{S, 0}$, the mean temperature $T_{m, 0}$ and the center temperature $T_{C, 0}$ used to define the initial condition ( $A$ and $r_{d c}$ in equation (32)) are given in Table 3 as well as the selected ambient gas temperature $T_{\mathrm{amb}}$.

Fig. 7 shows that the sensitivity of $\varepsilon$ to $K$, and therefore the accuracy of the adjustment of $K$, varies from one case to another. For cases 1 and 3 (3-pentanone and $n$-decane), $\varepsilon$ varies much more with $K$ than in the case 2 (n-dodecane). It means that it is simply not reliable to use the experimental maps to fix the value of $K$ in case 2 . Calculations show that the Peclet number of an isolated droplet, undergoing the same conditions as case 2, would not exceed 20. This value is all the more low since it is likely to be fairly reduced in the case of interacting droplets. In the case of $n$-dodecane, the sliding velocity $U_{s}$ is very limited because of the liquid viscosity of this fuel which is the highest among all the tested fuels (Table 1).

In contrast, the accuracy of the adjustment of $K$ can be roughly estimated to 0.5 for both cases 1 and 3 . This is sufficient to get some
Table 4

Results from the adjustment of the friction coefficient. Reductions of the drag and friction coefficients $\left(C d / C d_{\text {iso }}\right.$ is calculated using equation (33)).

\begin{tabular}{llllllrr}
\hline & Fuel & $D_{\text {inj }}(\mu \mathrm{m})$ & $V_{0}(\mathrm{~m} / \mathrm{s})$ & $C_{0}$ & $C d / C d_{\text {iso }}(\%)$ & $C_{F} / C_{F, \text { iso }}(\%)$ & $P e$ \\
\hline Case 1 & 3-pentanone & 230 & 9.5 & 4.2 & 23.1 & 7.1 & 55 \\
Case 2 & $n$-dodecane & 180 & 5.3 & 2.3 & 10.2 & $0-100$ & $0-20$ \\
Case 3 & n-decane & 190 & 6.7 & 2.7 & 14.5 & 3.9 & 24 \\
Case 4 & 3-pentanone & 201 & 9.9 & 3.2 & 18.3 & 3.9 & 33 \\
Case 5 & Ethanol & 179 & 6.7 & 2.3 & 12.4 & 1.6 & 5 \\
Case 6 & Ethanol & 233 & 10.1 & 4 & 23 & 9.9 & 64 \\
Case 7 & Ethanol & 216 & 9.4 & 3.5 & 19.5 & 12 & 68 \\
Case 8 & Ethanol & 131 & 8.1 & 5 & 22 & 19 & 30 \\
Case 9 & Acetone & 197 & 9 & 3 & 17.2 & 4.7 & 30 \\
Case 10 & n-heptane & 208 & 8 & 3.1 & 17 & 5.5 & 20 \\
Case 11 & $n$-heptane & 198 & 10.6 & 3 & 17 & 4.0 & 20 \\
\hline
\end{tabular}

indicates that the assumed decomposition of the friction coefficient (7) is rather satisfactory to account for important aspects such as the effect of volatility through the parameter $\left(1+B_{M}\right)$ and the effect of viscosity through the factor $\mathrm{Re}^{2 / 3}$

Despite the differences that may exist between the friction coefficient and the drag coefficient (the second including the form drag force in addition to the friction drag force), it can be interesting to refer to results available in the literature concerning the drag coefficient $C_{d}$. Atthasit et al. [27] investigated the effects of droplet interactions on the drag coefficient in the case of monodisperse streams of ethanol droplets evaporating in the thermal boundary layer of a heated plate. They suggested evaluating the drag coefficient as followed:

$\left\{\begin{array}{l}C d / C d_{\text {iso }}=a \ln (C)+b \\ a=3.43 \times 10^{-5} \operatorname{Re}^{3}-1.359 \times 10^{-3} \operatorname{Re}^{2}+1.8753 \times 10^{-2} \operatorname{Re}+4.84016 \times 10^{-2} \\ b=-1.07 \times 10^{-5} \operatorname{Re}^{3}+2.106 \times 10^{-4} \operatorname{Re}^{2}+7.668 \times 10^{-4} \operatorname{Re}-1.00236 \times 10^{-2}\end{array}\right.$

valuable information about the effects of droplet interactions on surface frictions. For instance, it can be noted that small values of $K$ are found which seems to indicate that the friction coefficient is sharply reduced by the interactions. Since $C_{F} / C_{F \text {,iso }}=K / 12.69$, the reductions of the friction coefficient are respectively $93 \pm 2 \%$ $(K=0.9)$ for case 1 and $96 \pm 2 \%(K=0.5)$ for case 3 . Finally, for case 3 , the evolution of the theoretical temperature field obtained with the optimal value of $K$ is shown in Fig. 8 alongside the experimental maps already presented in Section 3. A good agreement can be seen between the experiment and the theoretical predictions.

The adjustment of $C_{F}$ was performed for a dozen of cases. Measurement requires perfectly stable and periodic droplet streams over a long distance from the nozzle; this limits the possible range of investigation with the injector in terms of Reynolds number and distance parameter. The only fuel, for which adjusting $K$ was not reliable was $n$-dodecane. Table 4 shows the results of these adjustments. Note that cases 7 and 8 refer to ethanol droplet streams already described in Ref. [11]. Optimal values of $K$ were lower in Ref. [11] due to a different definition of $U_{s}$ (the factor $1 / 32$ in equation (6) was replaced by $1 / 6 \pi$ and $\varepsilon$ was not used as an indicator to adjust $K)$.

Fig. 9 shows the ratio $C_{F} / C_{F, \text { iso }}$ as a function of the distance parameter $C$. As expected, $C_{F} / C_{F, \text { iso }}$ increases with the distance parameter, but it is still equal to about $20 \%$ when $C=5$. A correlation between $C_{F} / C_{F \text {,iso }}$ and $C$ is observed regardless of the nature of the fuel. This observation gives some credence to the previous assumption that $K$ would be a function of $C$ alone. This also in the domain $8 \leq \operatorname{Re} \leq 19$ and $2.5 \leq C \leq 16$.

The same trend can be observed for both $C_{F} / C_{F \text {,iso }}$ and $C d / C d_{\text {iso. }}$. The reductions due to aerodynamic interactions are roughly the same order of magnitude, although they are more important in the

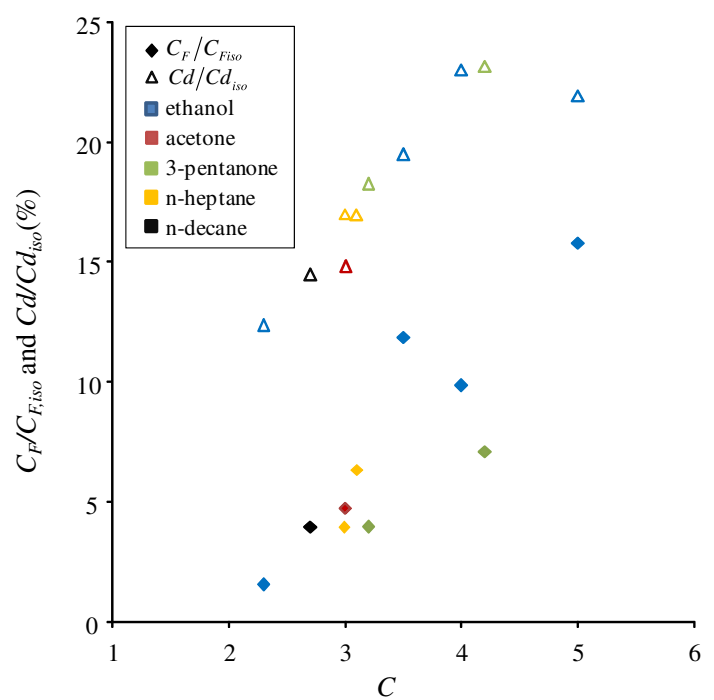

Fig. 9. $C_{F} / C_{F, \text { iso }}$ and $C d / C d_{\text {iso }}$ as a function of the distance parameter $C$. 
case of the friction coefficient. There is a gap of about $10 \%$ between the curves of $C_{F} / C_{F \text {,iso }}$ and $C d / C d_{\text {iso }}$ as a function of the distance parameter $C$. Fig. 9 provides therefore a convenient way to estimate the friction drag coefficient $C_{F}$ from the total drag coefficient $C d$ for droplets in strong interaction regime.

At this stage, several possible explanations can be suggested for

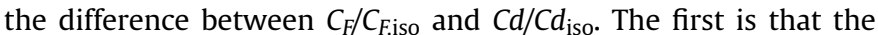
reduction of the form drag would be less important than that of the friction drag in the presence of strong aerodynamic interactions. This would be the result from the flow modification in the wake of the droplets. The second possible explanation refers directly to the remark at the beginning of Section 2. It deals with the possibility for the Marangoni effect to substantially slow down the internal flow. Although the Marangoni effect is not directly taken into account in this analysis, this effect is responsible for an additional surface stress. For an isolated droplet, Niazmand et al. [13] showed that the temperature of the droplet trailing edge is higher than that at the leading edge during most of the heating phase, resulting in a Marangoni stress that tends to slow down the velocity of the internal flow in opposition to the viscous drag. According to these authors, the Peclet number can be reduced transiently by an order of magnitude. However, there are no significant changes in the shape of the streamlines that remain very close to the Hill solution. The proposed analysis is therefore essentially valid even in the presence of the Marangoni effect, except that the parameter $C_{F}$ in equation (6) should be redefined as $F_{\text {frict }}+F_{\text {Marangoni }}=(1 / 2) \rho_{g} \Delta U_{\infty}^{2} S_{d} C_{F}$ to include the Marangoni force $F_{\text {Marangoni. In that case, the coefficient }}$ $C_{F}$ given in Fig. 9 does not correspond rigorously to the friction drag coefficient as it also includes the negative contribution of the Marangoni force in the droplet heating phase. Finally, the possibility of a bias due to some assumptions in the modeling is not totally excluded, for instance the fact of having considered a uniform temperature at the droplet surface in the simulations. Nevertheless, only advanced detailed simulations including these effects can determine what are the real contribution of the Marangoni force and the relative reduction of the friction and form drag.

\section{Conclusion}

Two-color laser-induced fluorescence has been used to characterize the temperature field within moving and interacting droplets in strong evaporation. The use of these fields to estimate the magnitude of the sliding velocity at the liquid-gas interface and hence the surface friction is not straightforward. A heat transfer model accounting for convection and conduction is required. This model is based on a number of assumptions, the strongest are that flow can be described by the streamlines of a Hill vortex and that the temperature surface is uniform. More refine but complex numerical simulations, including the resolution of the momentum and energy inside the droplet, coupled with a calculation of the outer flow, would avoid some potential biases in the modeling and give some valuable insights into the possible contribution of the Marangoni effect.

Nonetheless, we find with the previous assumptions that there is enough sensitivity to estimate the friction coefficient for most products that were tested with the notable exception of $n$ dodecane which is by far the more viscous liquid.

When the distance between the droplets is comparable with the droplet diameter, the reduction of the friction coefficient appears to be very important. This reduction exceeds $80 \%$ compared to an isolated droplet for distance parameters less than 5 and it logically increases when decreasing the distance between the droplets. It is also rather similar to the drag reduction due to the droplet interactions, which could give, in first approximation, a simple way to account for the internal mixing in the calculation of dense sprays.

\section{Acknowledgment}

This work has been conducted in the framework of the ASTRA program, supported by CNRS and ONERA.

\section{References}

[1] G.A.E. Godsave, Studies of the combustion of drops in a fuel spray: the burning of single drops of fuel, in: Fourth International Symposium on Combustion, 1953, pp. 818-830.

[2] W.A. Sirignano, Fluid Dynamics and Transport of Droplets and Sprays. Cambridge University Press, 1999

[3] D.B. Spalding, The combustion of liquid fuels, in: fourth international symposium on combustion, 1953, pp. 818-830.

[4] C.H. Chiang, M.S. Raju, W.A. Sirignano, Numerical analysis of convecting, vaporizing fuel droplet with variable properties, International Journal of Heat and Mass Transfer 35 (1992) 1307-1324.

[5] W. Shwin-Chung, L. Ar-Cheng, Internal temperature distributions of droplets vaporizing in high-temperature convective flows, Journal of Fluid Mechanics 237 (1992) 671-687.

[6] R. Kronig, J. Brink, On the theory of extraction from falling droplets, Applied Scientific Research 2 (1951) 142-154.

[7] B. Abramzon, W.A. Sirignano, Droplet vaporization model for spray combustion calculations, International Journal of Heat and Mass Transfer 32 (1989) 1605-1618.

[8] M.K. Akbar, S.M. Ghiaasiaan, Modeling the gas absorption in a spray scrubber with dissolving reactive particles, Chemical Engineering Science 59 (2004) 967-976.

[9] S.M. Ghiaasiaan, D.A. Eghbali, Transient mass transfer of a trace species in an evaporating spherical droplet with internal circulation, International Journal of Heat and Mass Transfer 37 (1994) 2287-2295.

[10] G. Castanet, P. Lavieille, M. Lebouche, F. Lemoine, Experimental and theoretical investigation of the heating of combusting droplets in a linear stream, Combustion Science and Technology 177 (2005) 2395-2422.

[11] G. Castanet, F. Lemoine, Heat transfer within combusting droplets, in: Proceedings of the Combustion Institute, 2007, pp. 2141-2148.

[12] R. Clift, J.R. Grace, M.E. Weber, Bubbles, Drops, and Particles. Academic, New York, 1978.

[13] H. Niazmand, B.D. Shaw, H.A. Dwyer, I. Aharon, Effects of Marangon convection on transient droplet evaporation, Combustion Science and Technology 103 (1994) 219-233.

[14] D.L.R. Oliver, J.N. Chung, Flow about a fluid sphere at low to moderate Reynolds numbers, Journal of Fluid Mechanics 177 (1987) 1-18.

[15] W.H. Chou, G.M. Faeth, Temporal properties of secondary drop breakup in the bag breakup regime, International Journal of Multiphase Flow 24 (1998) 889-912.

[16] B. Frackowiak, G. Lavergne, C. Tropea, A. Strzelecki, Numerical analysis of the interactions between evaporating droplets in a monodisperse stream, International Journal of Heat and Mass Transfer 53 (2010) 1392-1401.

[17] A.Y. Tong, W.A. Sirignano, Multicomponent droplet vaporization in a high temperature gas, Combustion and Flame 66 (1986) 221-235.

[18] G.L. Hubbard, V.E. Denny, A.F. Mills, Droplet evaporation: effects of transients and variable properties, International Journal of Heat and Mass Transfer 18 (1975) 1003-1008.

[19] M. Renksizbulu, M.C. Yuen, Numerical study of droplet evaporation in a hightemperature stream, Journal of Heat Transfer 105 (1983) 389-397.

[20] G. Castanet, A. Delconte, F. Lemoine, L. Mees, G. Grehan, Evaluation of temperature gradients within combusting droplets in linear stream using two colors laser-induced fluorescence, Experiments in Fluids 39 (2005) 431-440.

[21] G. Castanet, P. Lavieille, M. Lebouche, F. Lemoine, Measurement of the temperature distribution within monodisperse combusting droplets in linear streams using two-color laser-induced fluorescence, Experiments in Fluids 35 (2003) 563-571.

[22] P. Lavieille, F. Lemoine, G. Lavergne, J.F. Virepinte, M. Lebouche, Temperature measurements on droplets in monodisperse stream using laser-induced fluorescence, Experiments in Fluids 29 (2000) 429-437.

[23] V. Depredurand, P. Miron, A. Labergue, M. Wolff, G. Castanet, F. Lemoine, A temperature-sensitive tracer suitable for two-colour laser-induced fluorescence thermometry applied to evaporating fuel droplets, Measurement Science and Technology 19 (2008).

[24] P. Lavieille, F. Lemoine, M. Lebouche, Investigation on temperature of evaporating droplets in linear stream using two-color laser-induced fluorescence Combustion Science and Technology 174 (2002) 117-142.

[25] G. Castanet, M. Lebouche, F. Lemoine, Heat and mass transfer of combusting monodisperse droplets in a linear stream, International Journal of Heat and Mass Transfer 48 (2005) 3261-3275.

[26] V. Depredurand, G. Castanet, F. Lemoine, Heat and mass transfer in evaporating droplets in interaction: influence of the fuel, International Journal of Heat and Mass Transfer 53 (2010) 3495-3502.

[27] A. Atthasit, N. Doue, Y. Biscos, G. Lavergne, Influence of droplet concentration on the dynamics and evaporation of a monodisperse stream of droplets in evaporation regime, in: First International Symposium on Combustion and Atmospheric Pollution, 2003. 